\title{
Financial Analysis of an Illumination Retrofit for Regional Aircraft
}

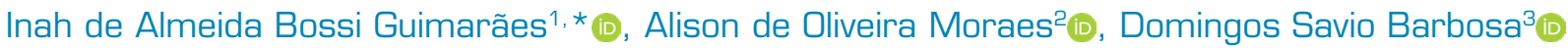

\begin{abstract}
1.Departamento de Ciência e Tecnologia Aeroespacial - Instituto Tecnológico de Aeronáutica - São José dos Campos/SP Brazil. 2.Departamento de Ciência e Tecnologia Aeroespacial - Instituto de Aeronáutica e Espaço - Divisão de Eletrônica - São José dos Campos/SP - Brazil. 3.Embraer - Interior System - São José dos Campos/SP - Brazil.
\end{abstract}

*Corresponding author: inah.almeida@engenharia.ufjf.br

\begin{abstract}
The light-emitting diode (LED) is the main lighting solution currently adopted in aircraft cabin lighting. This technology offers high durability, energy efficiency and luminosity, bringing plenty of benefits for being utilized in an aircraft. Despite its high initial price, it has many advantages, and the operational cost is lower than other types of illumination, such as fluorescent lamps. Additionally, LED illumination may improve the flight experience, making it more comfortable and pleasant for the passengers. Aircraft that have fluorescent lamps in their cabin should consider the possibility to make an illumination retrofit to LED lamps, because weight is directly correlated to the fuel consumption and operational costs and the energy economy can bring new improvements to the aircraft. The objective of this work is to evaluate the economic viability of the LED system installation compared with a fluorescent lamps illumination system in a regional aircraft. The study of the data shows that the usage of LED lights could save up to $68 \%$ of the energy in a month compared to conventional fluorescent lamps in the ceiling lights and reduce by $38 \%$ the monthly operational costs of illumination. The payback period is 4.2 years according to the proposed system.
\end{abstract}

Keywords: LED; Lighting; Energy saving; Payback; Replacement lamps.

\section{INTRODUCTION}

The aircraft lighting sector is expected to sustain significant growth in the coming years and one of the driving forces is the use of light-emitting diodes (LED) for interior cabin lighting. The increasing focus on enhancing passenger experience and the trend use of LED lights are major reasons for the growth in the aftermarket segment of the aircraft lighting field (Markets and Markets 2020). Besides that, in the aircraft area, there are challenges of increasing fuel economy, reducing weight, increasing capacity and improving customer experience. These challenges are creating space for innovation in lightweight products, smart lighting and energy-efficient equipment that resumes in the LED lighting, with less mass and less energy consumption.

Currently, aircraft cabin illumination, using LED lamp, supports designers to make an aircraft more comfortable for passengers. Modern aircraft cabin lighting has a pre-programmed setting of colors and intensity of light for specific flight

Received: Nov. 162020 | Accepted: Jan. 122021

Peer Review History: Double Blind Peer Review.

Section Editor: Ana Morais

This is an open access article distributed under the terms of the Creative Commons license. 
phases and time zones. Due to these settings, during sunset, sunrise and land or departure, the passengers can better enjoy the ambient and the overall flight experience. This system can help passengers overcome the fatigue of long-distance travel. Another strategy to increase passengers' comfort is to set a mix of colors that make them feel more relaxed in frightening moments, such as landing and departure. These are examples of how important lighting is in the cabin environment and justify the use of LEDs.

Until the early 2000s, the lamps used in aircraft were typically the fluorescents, incandescent and halogens. With the development of white LEDs, aircraft makers start to implement LED lamps in their aircraft (Escobar 2005). After 2010, aircraft started using mostly LED for their interior cabin illumination because it brings many benefits, as aforementioned. Given that an aircraft, on average, is operable for about 30 years before it has to be retired and some aircraft today are flying using old fashion illumination, it is convenient to analyze whether the upgrade of its illumination would be profitable and reasonable.

Additionally, the regional aviation market is a strategic and profitable sector where regional carriers typically operate aircraft with a seating capacity ranging from 20 to 130 seats, on short to medium-haul routes. By the end of 2015 , the regional aviation world fleet comprised about 9000 units more than $33 \%$ of the worldwide commercial fleet and performed over $40 \%$ of total commercial flights (and $25 \%$ of total flight hours). In the next 20 years, regional air traffic is expected to triple at an average yearly rate of 6\% (Clean Sky 2020). An important aspect is that a significant amount of this sector operates aircraft with old fashion illumination. This opens up an opportunity to provide services by offering an illumination retrofit. This retrofit consists of change in the fluorescent illumination of the aircraft cabin to LED lamps for regional aircraft.

This work presents an evaluation of the operational cost of the current lighting system in a regional aircraft and proposes a new lighting design to achieve the optimal use of energy and lower the operational costs. The study aims to evaluate if the usage of LED lights could provide significant energy saving compared to the conventional fluorescent lamps in the ceiling lights of a regional aircraft, maintaining an approximate amount of illuminance in the cabin and not changing the position and size of the lamps. The payback period is estimated based on the investment costs and the difference between the operational costs of both types of illumination, fluorescent and LED lamps, in order to verify if the illumination retrofit would be recommended, according to aircraft life span.

The next section presents aspects of the aircraft interior lighting, showing the type of illumination inside an aircraft, the main advantages of using LED on the aircraft and describes the lighting methods that have been utilized in the aircraft cabin. The ensuing section analyzes the methodology of the retrofit for a regional aircraft, demonstrating the feasibility analysis of replacing T8 tubular fluorescent lamps with LED tubes for the cabin environment of a regional aircraft. The conclusions present the reason this analysis was made, the benefits that the retrofit can bring to the aircraft, the amount of saving energy and the calculated payback period to help the decision of the viability of the retrofit.

\section{ASPECTS OF AIRCRAFT LIGHTING}

\section{Aircraft light}

Lighting is essential in the aircraft for various tasks, such as safety, operational needs, servicing and the comfort of passengers. These lights may be grouped in four areas: flight compartment (cockpit), passenger cabin, exterior and servicing (cargo and equipment bays). This work focuses on the light of the cabin of commercial aircraft, the place occupied by the crew members and passengers. In the cabin interior, there are different kinds of lights, including:

- Dome lights: located on the ceiling providing general illumination for the entire main cabin, one of the main light sources within the aircraft cabin. Usually, the crew operates the lighting from the flight attendant panel located next to the door. Figure 1a shows an example of cabin interior and the dome light composed by the spots in the middle of the aircraft. 
- Wash lights: strips located above the sidewall illuminating the ceiling and the side of the aircraft; these are the main light sources of the cabin. The location and amount of these strips depend on aircraft design and customization. These lights are operated with the dome light by the crew, from the flight attendant panel. In Fig. 1a, the wash lights are the lights at the ceiling and in the sidewalls.

- Passengers reading lights: it is an independent lighting system at the top of the passenger seat and controlled from individual seat controls so that passengers can read when the cabin lights are off. Figure $1 \mathrm{~b}$ shows one example of the individual reading lights, near the air gasper, of the E-jets E2 Embraer aircraft.

- Warning signs light: these signs are usually activated by the pilots and must be lighted during take-off, landing, turbulence events and when the pilots deem to be necessary. For example, seat belts or no-smoking signs. Figure 1c shows the cabin light of Embraer E-Jets E2 aircraft.

- Spotlights: small lights for decoration, usually used to illuminate the cross aisles, galley and entry areas. Figure $1 \mathrm{~d}$ shows one example of spotlights.

- Flexible light strips: these are decoration lights that illuminate surfaces to enhance, for example highly customized bar or lavatory monuments. Figure 2 a shows an example of this flexible light strip under the mirror.

- Lavatory lights: lights utilized in the lavatory. Figure $2 \mathrm{~b}$ shows the lavatory lights in the ceiling of the toilet.

- Emergency lights: for emergency evacuation purposes. The emergency lights are composed of floodlights in the door areas, an "EXIT" sign located adjacent to the emergency exits that must be clearly visible, and floor path lighting to provide visual identification of escape routes along the cabin aisle floor in case of dense smoke. The emergency lights are required by the authorities to comply with Regulamento Brasileiro de Homologação Aeronáutica (RBHA) from Agência Nacional de Aviação Civil (ANAC), title 14 of the Code of Federal Regulations (CFR) from Federal Aviation Administration (FAA) and Certification Specifications (CS) from European Union Aviation Safety Agency (EASA) 25,812 requirements. These lights shall be independent of the main electrical system. Panels (c) of Fig. 2 show the floor proximity lighting aids airplane evacuation under dark or smoky conditions and the emergency lights of a Boeing aircraft.
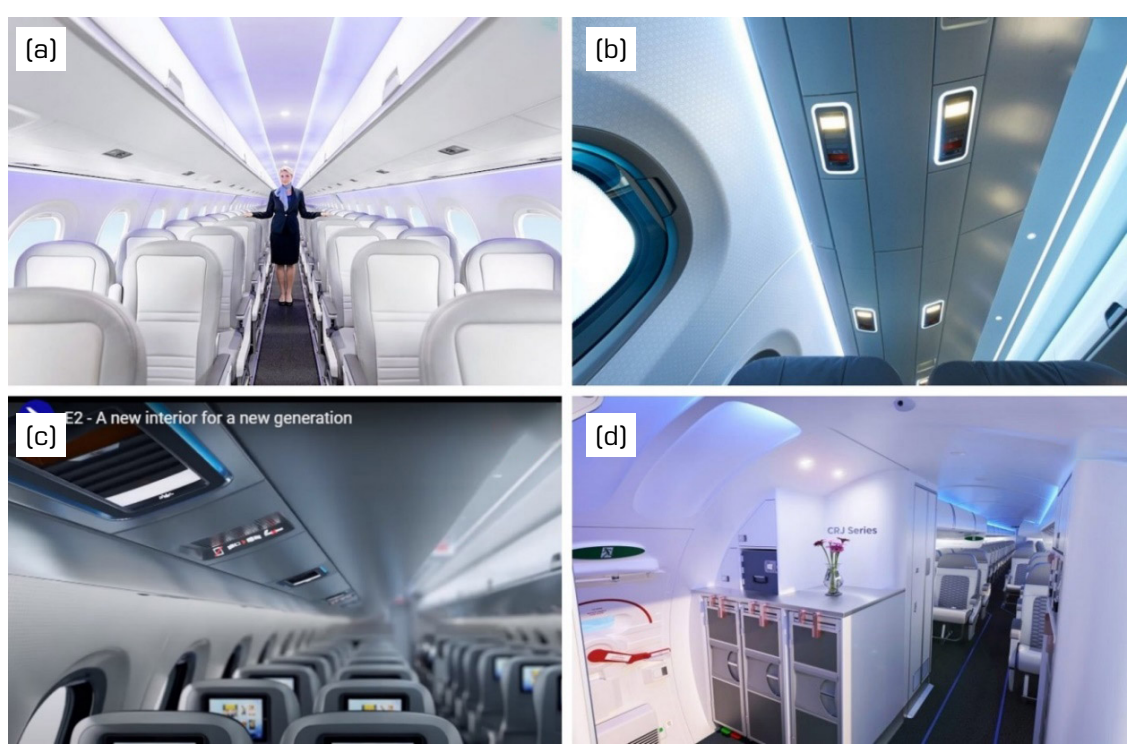

Figure 1. Different types of cabin interior lights. (a) Dome and wash lights of the Embraer E-jets E2 (Eden 2020). (b) Reading lights of the E-jets E2 Embraer (Airlines IATA 2014). (c) Warning sign lights of the E2 aircraft from Embraer (Embraer 2020). (d) Spotlights in the galley area of the CRJ Series of Bombardier (Aeroflap 2018). 

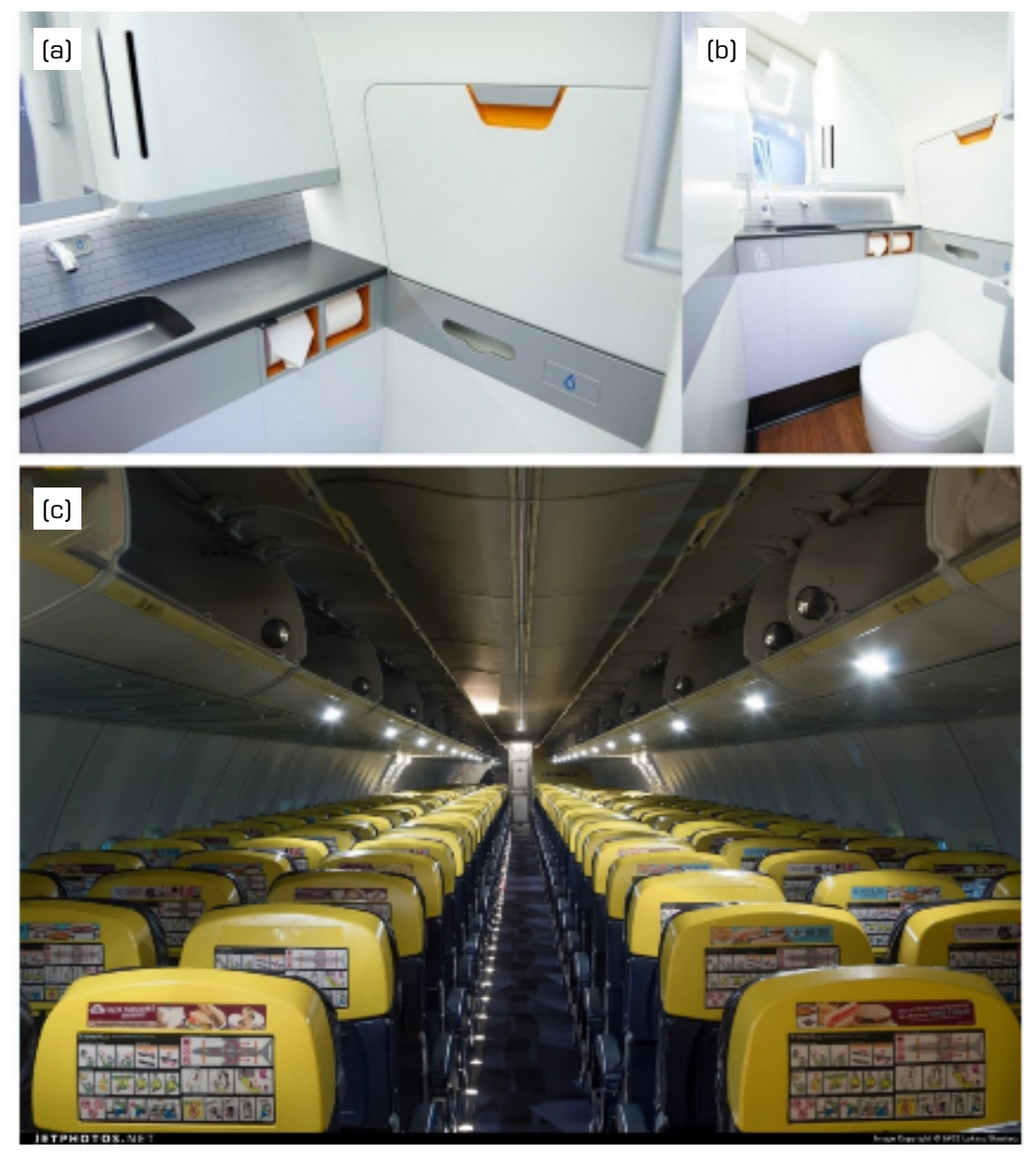

Figure 2. Aircraft cabin interior lights. Panel (a): Flexible light strip under the mirror. Panel (b): Lavatory lights of E-jets E2 of Embraer (Airlines IATA 2014). Panels (c): Emergency light of a Boeing aircraft (Stawiarz 2014).

\section{Advantages of the LED lamp in the aircraft}

This subsection describes the main benefits of using LED lighting. Among the many advantages of LED for cabin illumination, the following aspects are worth mentioning:

- Maintenance: LED lamps may have life cycles of 6 times compared with fluorescent lamps (Nuhoglu 2016) and are more reliable, requiring less maintenance, reducing aircraft ground time (STG 2017) and decreasing intervals between unscheduled repairs.

- Weight: this is one of the most important factors on an aircraft, because it is related to fuel cost. Light-emitting diodes are very small, making it low weight. Light-emitting diode lamps may be only $1 / 3$ of the weight of incumbent systems helping to reduce fuel burn (STG 2017).

- Environment: LED systems do not contain any toxic substance and are safe for disposal, unlike fluorescent lamps that contain mercury and must be properly recycled. Besides, the long-life span of LEDs diminishes the breakdowns and eliminates the need of changing the bulb often.

- Robust: LEDs material is resistant to shock and vibration, events that occur frequently in the environment of an aircraft.

- Easily dimmable: LED light is easily dimmable by controlling its electrical current. In aircraft, it helps in the interior design project to change the brightness of the lights in long flights so the passenger can sleep.

- Energy-efficient: LED may save energy up to 50\% compared with fluorescent lamps (Nuhoglu 2016). 
- Design flexibility: LED may be produced in any shape or size. It can be dimmable and its color may be set and changed, creating different environments, which is great for passenger experience.

- Generate less heat: Compared to other light technologies, LED dissipates little heat, which is good for the comfort of the passengers and the environment of the aircraft.

\section{Lighting schemes for aircraft cabin}

The LED lights provide many lighting methods that have been utilized in the aircraft cabin. This lighting scheme helps the passengers relax and feel more comfortable during the flight.

The mood lighting is the latest trend in the aircraft interior lighting market and it may simulate a morning sunrise, a bright fresh afternoon or a cool evening by continuous changing of colors using LED. It artificially alters the perception of how long a day or a night is, aiming to reduce the likelihood of jet lag. Another good way that may help to reduce jet lag is to use artificial lighting to mimic and match the destination environment. Figure 3a shows a type of mood light used by American Airlines in the 777 aircraft by Boeing. The aircraft lighting design may, for example, create combinations of colors that remind natural or calendar events, such as Aurora Borealis, Chinese New Year and several other festive scenarios.

The preset light is a specific light configuration designed to match a certain action, it uses a mix of colors and different light intensities. Examples of actions are boarding and deboarding time, dinner time, or sleep time. Figure 3b, c and d of show different types of illumination for distinct moments of the flight in the aircraft A380.
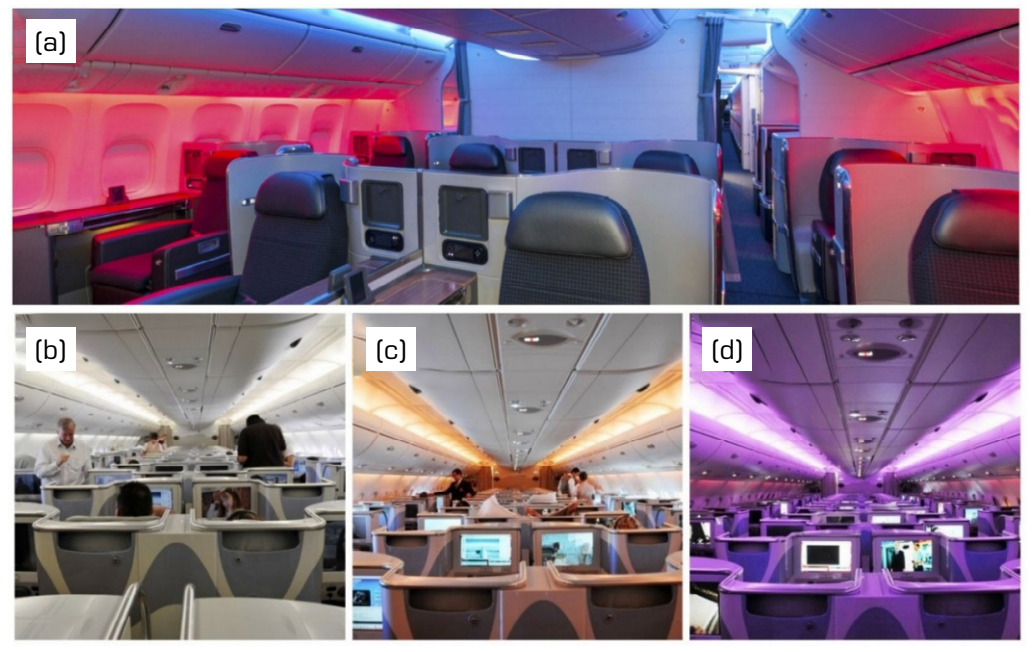

Figure 3. Lighting schemes in an aircraft cabin. (a) Mood lights in the cabin of Boeing 777 (Bachman 2015) and (b), (c) and (d) Airbus 380 boarding, dining, and sleep lightings, respectively (IflyA380 AIRBUS 2018).

\section{METHODOLOGY}

This section presents the methodology used to assess the feasibility of installing LED lighting. The design of a lighting system considers visual environmental requirements, quality of light, type of luminaire and application of the room. The redesign of the lighting installation focuses on the optimization of energy consumption and explores efficient ways to decrease the total cost of the aircraft operation.

Firstly, an aircraft was selected as a reference for the proposed analysis. Afterwards, generic fluorescent and LED tube lamps that typically may be utilized inside of an aircraft were also chosen. According to the chosen lamps, an individual analysis of the specifications provided by the manufacturer datasheet was performed considering the factors and values that are commonly 
needed in an illumination project. Then, an estimation based on the aircraft design was made to evaluate how many lamps should be used inside this aircraft. The number for LED and fluorescent lamps was defined to be the same because it is a retrofit analysis. Posteriorly, the investment cost and the maintenance fees were added. The comparison between the performances of these two distinct lamps inside of the aircraft and the ensuing conclusions were based on the payback period, i.e., in case of an acceptable payback period for the airlines, illumination retrofit would be recommended.

Figure 4 shows a flowchart of the methodology mentioned.

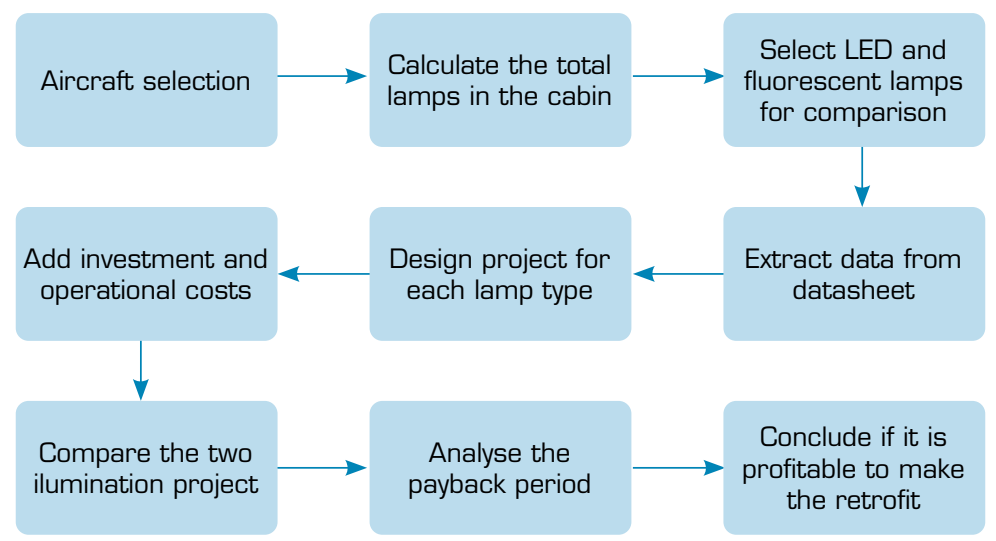

Figure 4. Flowchart of retrofit feasibility analysis methodology.

The selected aircraft to be analyzed by the proposed methodology is any general regional aircraft with more than 630 units delivered, which still has a solid order backlog of more than 150 aircraft, and is currently one of the most delivered aircraft. In this case, it was assumed that the aircraft could carry up to 88 passengers in a single-class high-density configuration. Another assumption is that, today, this aircraft uses fluorescent lamps. This is essential to estimate the payback period and to analyze the possibility to change the cabin lights for LED lamps. Figure 5 shows an example of a regional aircraft with characteristics similar to those assumed in this analysis.

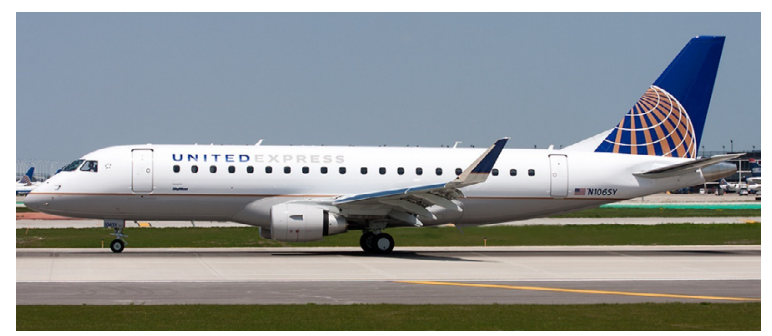

Figure 5. Regional aircraft (Brodkey 2014).

\section{Assumptions in the retrofit analysis}

The retrofit analysis in this work covers the wash light in the ceiling of the cabin of the area where the chairs are located. Inside of an aircraft, the ceiling light is distributed all along the length of the aircraft in the passenger area. For the regional aircraft model, the ceiling light is installed in four lines through the aircraft, in the sidewalls above the windows for each side and two more in the middle of the aircraft, near the bins.

In this analysis, the number of lights was assumed to be equivalent to the number of bins in the regional aircraft multiplied by four because of the four positions through the aircraft mentioned previously. Figure 6 shows the number of bins and lamps per line through the aircraft and Fig. 7 shows the four positions of the lamps indicated as green circles. In order to perform the analysis, each lamp must have a length of approximately $1.5 \mathrm{~m}$, which is the size of the bin. Therefore, the calculated number of lamps inside this regional aircraft is 40 . 


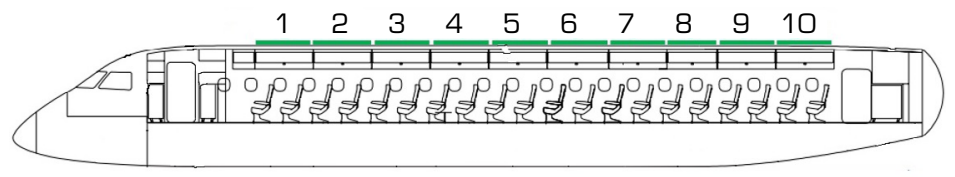

Figure 6. Number of bins in a regional aircraft. Adapted from Embraer (2020).

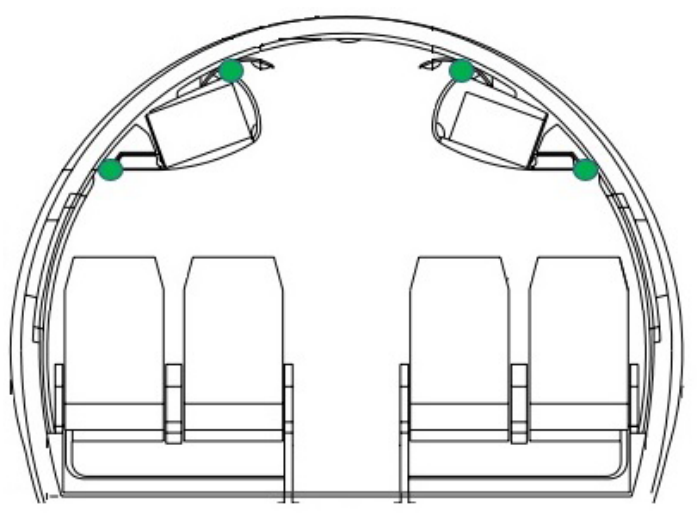

Figure 7. Position of the lamps in a regional aircraft. Adapted from Embraer (2020).

To an illumination retrofit design, the LED lamps must be the same number and size as the original fluorescent lamp, so the new lamps will be at the exactly same place as the old lamps, not harming the illumination design of the aircraft and not having places with dark spots. The lamps used in this comparison are of fluorescent type, as the current lighting system, and a LED lamp type that represents the retrofit lamp. The chosen lamps have characteristics very similar to the original lamps utilized in the wash light of the ceiling and walls of an aircraft cabin.

The lamps assumed in this study were the Sylvania 21916 - FO40/841/XP/ECO3 - T8s made by Sylvania (2021) and F60/HO - F60T12/HO made by Satco (2021), both of them are recessed luminaires for general lighting and are tubular T8 size. Figure 8 shows the lamps utilized in the calculations. The technical specifications of these lamps are described in Table 1.
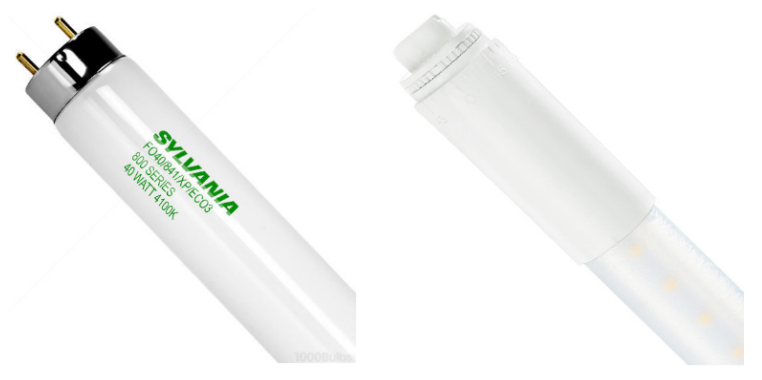

Figure 8. Fluorescent lamp on the left side and the LED lamp on the right side (Sylvania 2021), (Satco 2021)

Comparing the lamp models in Table 1 , it is possible to observe that:

- Both lamps have approximately similar luminous flux, but the power of the LED lamp is almost half of the fluorescent lamp. That is shown in the lumen/watt factor efficiency. This ratio indicates that the LED lamp is more energy-efficient, producing the same luminous flux with significantly less energy.

- The LED lamp has a higher expected life comparing to the fluorescent lamp.

- The fluorescent lamp has the additional power of the ballast that increases the total power, the LED lamp has the driver in it as well, but it is already included in the power of the lamp.

- The LED lamp does not contain any mercury, making it better for the environment. 
- The color rendering index (CRI) compares the color of an object under artificial light with the real color of that same object, under the natural light from the sun. It ranges from zero to one hundred. The CRI of the chosen lamps is above 80 . This is a satisfactory number to be utilized inside an aircraft.

The fluorescent lamp has negative resistance characteristics and therefore must be operated with ballast, which is a current limiting device (Dilaura et al. 2011). Eliminating it will save weight, space and will reduce maintenance costs. It may weigh close to $0.9 \mathrm{~kg}$ each. In this retrofit analysis, it was considered the weight of the ballast in the financial calculation, however, the removed volume of the ballast will be beneficial for the aircraft as well. For example, a Challenger 601 business jet has 16 to 18 ballast, thus, a reduction between 14.5 to $16.3 \mathrm{~kg}$ of the aircraft may be achieved, with the new space that may possibly transport another carry-on bag (Wynbrandt 2016).

Table 1. Technical data of the lamps used in the analysis. (Sylvania 2021) (Satco 2021).

\begin{tabular}{ccc}
\hline Property & Fluorescent Lamp & LED lamp \\
\hline Expect lifetime & $36,000 \mathrm{~h}$ & $50,000 \mathrm{~h}$ \\
\hline Power & $40 \mathrm{~W}$ & $24 \mathrm{~W}$ \\
\hline Ballast power & $35 \mathrm{~W}$ & - \\
\hline Luminous efficacy & $93.75 \mathrm{~lm} / \mathrm{W}$ & $129 \mathrm{Im} / \mathrm{W}$ \\
\hline Color rendering index & 85 & 83 \\
\hline Total lighting output & $3750 \mathrm{~lm}$ & $3100 \mathrm{~lm}$ \\
\hline Color temperature & $4000 \mathrm{~K}$ & $4000 \mathrm{~K}$ \\
\hline Length & $151.4 \mathrm{~cm}$ & $152 \mathrm{~cm}$ \\
\hline Mercury content & $5.5 \mathrm{mg}$ & - \\
\hline Weight lamp + ballast & $1102 \mathrm{~g}$ & $609 \mathrm{~g}$ \\
\hline Diameter & $2.54 \mathrm{~cm}$ & $2.54 \mathrm{~cm}$ \\
\hline
\end{tabular}

\section{RETROFIT ASSESSMENT}

\section{Performance comparison}

In this work, the total installed power $\left(P_{t}\right)$ was defined as the sum of the power of all devices installed in the ceiling lighting system. This is the power of the lamp added to the power of the ballast, multiplied by the number of units used ( $\mathrm{n}=40$ ). For the fluorescent lamp $P_{t f}$ is given by Eq. 1:

$$
P_{t f}=\mathrm{n}_{f} \times w=40 \times(40+35)=3000 W
$$

where $n_{f}$ is the total number of units and $w$ is total power consumption, in this case, $40 \mathrm{~W}$, according to Table 1 , with $35 \mathrm{~W}$ added because of the utilized ballast for each fluorescent lamp. For the LED case, with no need to use ballast, the total installed power $P_{t L}$ is given by Eq. 2:

$$
P_{t L}=\mathrm{n}_{L} \times w=40 \times 24=960 W
$$

Table 2 presents information about the wattage and the total power of each lamp. It is possible to verify that LED consumes the least amount of power. A saving of $2.04 \mathrm{~kW}$ may be attained in the new system, reaching $68 \%$ of savings in electric energy. 
Table 2. Wattage and the total power of each lamp.

\begin{tabular}{ccc}
\hline Power & Fluorescent lamp & LED lamp \\
\hline Per unit (W) & $40+35$ & 24 \\
\hline Total (W) & 3000 & 960 \\
\hline
\end{tabular}

It is worth noticing that, in the initial phase of an aircraft project, all the energy that is used in the aircraft is estimated and considering this the engine and electricity generator of the aircraft are chosen. After the project phase, when the aircraft is already certified and flying, if the energy consumption is reduced this will not affect the current costs of the aircraft, because the same initial power is still being generated. Despite this, the reduced power brings some benefits to the aircraft. The aircraft is in constant modification, always accumulating service bulletin (SB). An SB is a notice to an aircraft operator from a manufacturer informing him/her of product improvement. It may be a safety-related or improvement of a product. In the case of an optional SB, it may bring some benefits to the passenger experience and make the aircraft more competitive compared to the newer versions. For example, older aircrafts did not have outlets in the cabin area, but nowadays is a very important item for the passenger. Most of the modifications in an aircraft needs energy, so the saved energy may be used for future modifications in the aircraft. This saved energy will not be considered during operational costs calculation, but brings benefits anyways.

\section{Cost evaluation}

After the power consumption estimation, the next step is the cost calculation. This analysis is important to check the viability of the retrofit. The evaluation will be divided into initial and operational costs. To compare the cost of both lamps, the use of the light was discriminated monthly.

For the cost of the project and installation, the salary of two workers in Brazil was considered, one engineer and one mechanic, for project adaptation and installation. In this context, the installation was estimated in one week and the project in one month, with a total cost of around US\$ 4,000.00. The investment cost (IC) is the sum of the acquisition costs of all the equipment that makes up the lighting (e.g., lamps, fixtures, ballast, transformers, ignitors and wiring) plus labor costs for professionals involved, since the preparation of the project to final installation. Taking the project and installation cost, considering the price of each LED lamp, according to Table 3 (US\$ 120), for a total of 40 units, the $\mathrm{IC}$ is calculated in $\mathrm{Eq} 3$ :

$$
I C=4000+(40 \times 120)=U S \$ 8800
$$

Table 3 presents the IC for each type of lamp. It is adequate to mention that, in Table 3, the IC is only considered for the LED lamp case because it is a retrofit analysis, the fluorescent lamp is already in the aircraft.

Table 3. Investment cost for each type of lamp.

\begin{tabular}{ccc}
\hline Investiment & Fluorescent lamp & LED lamp \\
\hline Cost per unit (US\$) & 15 & 120 \\
\hline Cost per project and installation (US\$) & - & 4000 \\
\hline
\end{tabular}

Figure 9 decomposes the two factors of IC, namely, material (lamps) and service (project and installation).

The operational cost (OP) is the sum of all costs presented after the complete installation of the lighting system, concentrated on the maintenance costs of lighting conditions of the project, the monthly cost of acquiring new units of a lamp when burning and the cost of the weight that was saved by the change of illumination. A commercial aircraft typically works about $12 \mathrm{~h}$ a day and most of the time the ceiling lights are turned on, being on the ground or flying. Based on this, the time, $t$, of hours used by month will be: $t=12 \times 30=360 \mathrm{~h} /$ month . 


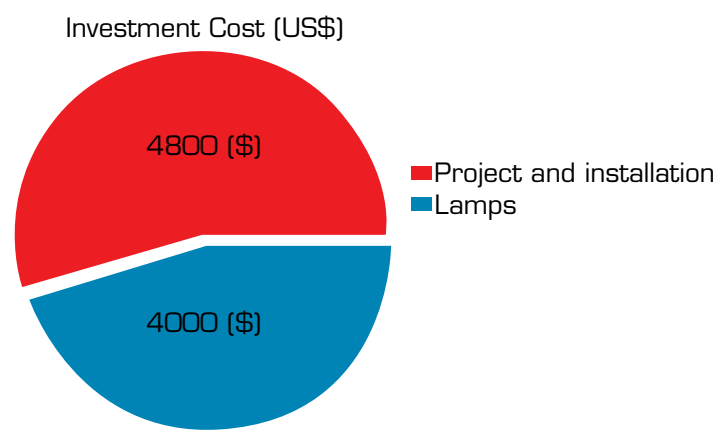

Figure 9. Distribution of the proportion of the two factors of the investment cost.

The monthly cost of acquiring new units of lamp replacement when useful lifetime is completed was calculated from the multiplication of the monthly hours of lamp usage, the number of lamps and the price of the lamp divided by its useful life and the lumen depreciation index (OSRAM 2014). The monthly cost for fluorescent $\left(C_{l f}\right)$ and LED $\left(C_{l L}\right)$ lamps are, respectively represented in Eqs. 4 and 5:

$$
\begin{gathered}
C_{l f}=\frac{n_{f} \times t \times c_{f}}{p_{f} \times \eta_{\mathrm{Lf}}}=\frac{40 \times 360 \times 15}{36000 \times 0.82}=U S \$ 7.32 \\
C_{l L}=\frac{n_{L} \times t \times c_{L}}{p_{L} \times \eta_{L L}}=\frac{40 \times 360 \times 120}{50000 \times 0.80}=U S \$ 43.2
\end{gathered}
$$

where, $n_{f}=n_{L}$ is the total number of lamps, $t$ the monthly usage time, $C_{f}$ and $C_{L}$ the fluorescent and LED lamps cost, respectively. Also, $P_{f}$ and $P_{L}$ are the expected lifetime for fluorescent and LED lamps. The term $\eta_{\mathrm{L}}$ (additional $f$ and $L$ subscripts determine the nature of the lamp in each equation) is the lumen depreciation and it occurs with all types of electric light sources. The lumen depreciation is defined as the loss of the original light output of the lamp or fixture that occurs over time. The LED lamp has lumen depreciation, at the end of its life, of $20 \%$ and the fluorescent lamp has depreciation, at the end of its life, of $18 \%$ (Principi and Fioretti 2014).

Although the LED illumination system has a longer life cycle, the lamp cost is much higher than the fluorescent one. This is the reason why the operational cost of lamp repositions for LEDs will be higher.

The monthly cost of labor required to perform the maintenance, including the replacement of lamps or troubleshoot, is given according to the hourly wages of the respective professional. The justification of the values chosen here is that the professional will have to change more fluorescent lamps than LED lamps. Additionally, fluorescent lighting systems that require ballasts are innately more time consuming to troubleshoot, because an inoperative light may be caused by a burned-out lamp or a problem in the ballast (Wynbrandt 2016). Moreover, the fluorescent lamp must be recycled at the end of life and requires special disposal, hence some additional cost is expected to be added.

The monthly cost of weight reduction was calculated according to the value of the weight items cost of one 737-800 (Stone 2017). This weight must be calculated because every item onboard makes the plane heavier, increasing fuel usage and the overall operational cost. It states that the cost of the weight for one year is US\$ 68.34 per kg, utilizing typical U.S and European flight conditions of three major carriers (United, American and Ryanair) over a normal day. This value may be utilized in this calculation, despite the difference of the engine and the size of the plane. Equations 6 and 7 calculate the cost of the weight.

$$
\begin{gathered}
C_{w f}=\frac{n_{f} \times \rho \times \kappa_{f}}{m}=\frac{40 \times 68.34 \times 1.102}{12}=251.05 \\
C_{w L}=\frac{n_{L} \times \rho \times \kappa_{L}}{m}=\frac{40 \times 68.34 \times 0.609}{12}=138.74
\end{gathered}
$$


where $\rho=$ US $\$ 68.34$ is the aforementioned cost of the weight per year, $k_{\mathrm{f}}$ and $k_{L}$ are the weight of each lamp plus ballast (for fluorescent and LED lamps, respectively), according to Table 1 . Both equations have a factor $m=12$ to achieve the monthly value. The total operational cost of the two types of illumination is presented in Table 4 and summarized in Fig. 10. Both (Table 4 and Fig. 10) reveal that a reduction of the operational cost by $38 \%$ may be accomplished.

Table 4. Operational cost by month for each type of lamp.

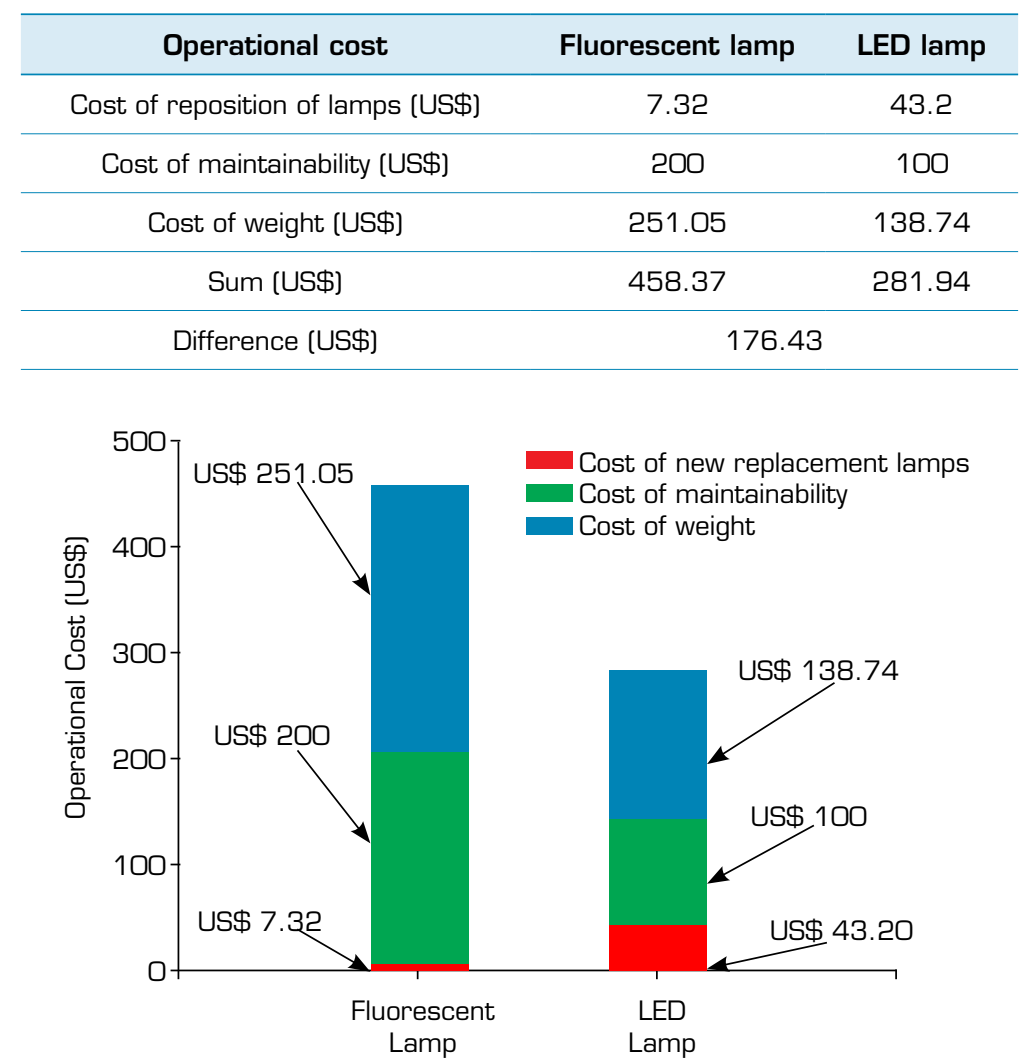

Figure 10. Operational cost for each type of lamp monthly.

The comparative analysis of the two lighting systems (to establish the viability of a retrofit) considers the new illumination investment and both operating costs. Usually, the use of a better energy-efficient illumination system leads to higher investment but provides savings at operational cost.

For the comparison of the expenses of the two lamps and obtainment of the payback, it is necessary to define the payback time, which is calculated based on the investment cost and the difference in the operational cost. This method is often applied in comparison design and as an approximation to the amount of time required to pay off an investment. However, it does not consider the time value of money, i.e., the fact that a given monetary amount may be worth more today than at some point in the future and vice-versa. According to Dilaura et al. (2011), this value is given by Eq. 8:

$$
\text { Payback time }=\frac{(\mathrm{IC} / \Delta)}{\mathrm{m}}=\frac{(8800 / 176.43)}{12}=4.15 \text { years }
$$

where $\Delta$ is the difference in the operational cost of the LED lamp in comparison to fluorescent one in Table 4 and $m$ is total months of the year. Table 5 shows the investment costs plus the operational costs for each type of illumination. The return of investment is about 4.2 years. This means that the retrofit is highly indicated for aircraft with more than 4.2 years of working life. Under this condition, the operational cost savings will recover all the money invested. 
Table 5. Sum of the investment and operational costs for each type of illumination over the years.

\begin{tabular}{ccc}
$\begin{array}{c}\text { Time } \\
\text { (year] }\end{array}$ & $\begin{array}{c}\text { Operational cost for fluorescent lamp } \\
\text { [US\$] }\end{array}$ & $\begin{array}{c}\text { Investment + operational cost for LED lamp } \\
\text { [US\$] }\end{array}$ \\
\hline 1 & 5500.37 & 12183.24 \\
\hline 2 & 11000.74 & 15566.48 \\
\hline 3 & 16501.11 & 18949.72 \\
\hline 4 & 22001.49 & 22332.96 \\
\hline 5 & 27501.86 & 25716.20 \\
\hline
\end{tabular}

By comparing the expenditures of the two lamps after a period of five years it is possible to notice a save of US\$ 1785.66. It can be seen that the tubular LED lamp would already be more worthwhile than the tubular fluorescent due to its lower energy consumption and operational expenses.

The analysis of investment and operational costs over time is presented in Fig. 11 in the form of lines for LED (blue line) and fluorescent (orange line) lamps. The initial investment amounts are added to the monthly operational expenses. The intersection point of the lines occurs in 4.2 years, indicating the equalization of these costs. After this point in time, the LED type of illumination turns to a cost lower than the fluorescent type. The accumulated cost savings along 30 years for a project of 40 lamps would reach US\$ 54,714 .

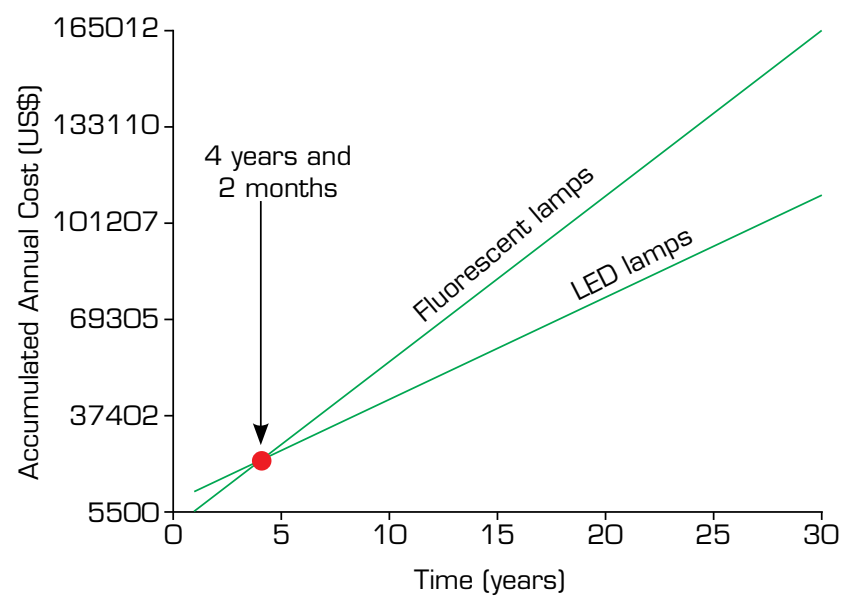

Figure 11. Analysis of the accumulated costs for fluorescent (orange line) and LED (blue line) lamp over time. After 4.2 years, the LED lamp becomes progressively more indicated.

\section{DISCUSSION}

The retrofit study case shows the advantages of using LED in the aviation environment as well as its financial benefits. The calculations shown here have some estimates, hence, is adequate to mention that in case of different initial values results may be affected. Therefore, this topic will discuss what would be the changes in the output in case of distinct input values.

If the aircraft flying time per day was assumed to be $8 \mathrm{~h} /$ day instead of the assumed value of $12 \mathrm{~h} /$ day, thus implying an amount of $240 \mathrm{~h}$ as the total hours a month, that would impact the cost of the lamp reposition. The difference in the operational cost would increase slightly, from US\$ 176 up to US\$188. Because the difference in the operational cost is higher, the payback period is lower and it is 3.89 years. In the case of a $4 \mathrm{~h} /$ day flight time, the payback period would be 3.66 years. Given that the cost of the reposition of the lamp is an important factor in the operational costs, the outcome would be slightly affected, making it faster to achieve the payback period. 
The analysis was made utilizing a small regional airplane with only one aisle, but if this was applied to a larger scale, in a bigger airplane, the LED lamp presents itself as a much more economical alternative than the fluorescent illumination. A big aircraft with two aisles would use twice the number of lamps for both types of illumination. This modification would considerably affect all the factors of the project. The maintainability cost would increase as well because of the number of lamps and the size of the aircraft. Doubling the number of LED and fluorescent lamps and increasing by $50 \%$ the maintainability cost, the payback period would be of 3.74 years. The difference in the operational cost increases, but the initial cost increases as well. So the size of the aircraft and the number of lamps have a very small impact on the payback time.

The LED lamp nowadays is expensive but its price tends to decrease in the next years. Hence, if the LED lamp was $40 \%$ lowpriced (i.e. instead of US\$ 120 was US\$ 72) the payback period would be of 2.96 years. This change would impact the initial cost and the cost of the lamp's reposition as well and would decrease substantially the payback period.

In case the project and installation cost are increased by 50\%, it would rise from US\$ 4000 to US\$ 6000, the payback time would be 5.10 years. If the maintenance costs are lowered by $50 \%$ for both illumination types, the payback time would be 5.80 years. Even in these cases, both payback periods are not high and would still make the retrofit project viable depending on the remaining time of the aircraft. If the maintenance of the LED and fluorescent lamps have the same cost, this would increase the payback value to 9.59 years, which could be a long payback time depending on the aircraft. This occurs because the difference between the operational cost would be reduced drastically.

A payback period of approximately six years is considered worthwhile to the retrofit project, because an aircraft life cycle is approximately thirty years, so the payback period should be lower than the remaining years of operation of the aircraft, as stated earlier. It is important to remember that the LED type of illumination benefits the passenger experience and comfort, and also the implementation of new SB due to the energy consumption savings. So even if the remaining life of the aircraft is a little lower than the payback time, the retrofit project could still be a good option.

By analyzing all these different assumptions, it is possible to verify the robustness of the retrofit results. Even in case that some of the initial assumed values needed to be adjusted, this would only cause a small impact on the payback time and hence, the illumination retrofit would still be profitable and recommended.

\section{CONCLUSION}

The flexibility of LED opens new possibilities for design, brand identification and customer satisfaction. In an incredibly costcompetitive industry, these are significant drivers and manufacturers are moving towards LED as a solution, both for new models and for replacement lighting on existing aircraft.

To analyze the retrofit viability and their financial costs for a regional aircraft, this study was made using the information of a regional aircraft, generic LED and fluorescent lamps. A comparison between the two lighting projects for each type of illumination technology utilized in the cabin ceiling of an aircraft was performed. Two types with near luminous flux, different power and the same quantities of lamps were selected. The total power consumed decreases from 1080 to $346 \mathrm{kWh} / \mathrm{month}$, which is an economy of $68 \%$ in energy. The lighting energy consumption in an aircraft flying $12 \mathrm{~h}$ /day may be reduced by $8813 \mathrm{kWh}$ per year. This energy economy brings a very important benefit for the aircraft, allowing future modifications of it, that may be essential or improvement of any type, making the aircraft better for the users.

The financial study shows that the LED lamp has a higher investment cost, due to the price of the lamps, project and installation, but the operational cost is lower because of the reduced cost of maintainability, replacement of lamps when burn and weight, being weight and maintenance cost the most important parameters. The LED tube lamp working $12 \mathrm{~h} /$ day along 365 days a year would have a payback return time in 4.1 years and the save cost in 30 years would be US\$54,714. Therefore, the retrofit would be a viable alternative to a regional aircraft, if it has a remaining operational life longer than 4.1 years.

However, it is not possible to state that this exchange is valid for all manufacturers and models of fluorescent and LEDs on the market, as there is a huge variety of powers, prices and durability. It must always be noted that, in addition to the price, other aspects need to be taken into account. Among these aspects, the luminous flux similarity between fluorescent and LED lamps, the power in the LED lamp must be lower (consequently, with less energy consumption), the size of the lamp and the socket must be compatible, the color temperature must match and the color reproduction indices must be adequate. 
In the discussion section, the robustness of the calculation method applied in this study was analyzed, varying some of the initial inputs to a certain the conclusion. It can be noted that modifying the flight hours per day and the size of aircraft would cause a slight reduction in the payback period. Decreasing the price of the LED lamp by $40 \%$ would reduce the payback period from 4.15 years to 2.96 years. If the investment cost was increased by $50 \%$, the payback period would be 5.10 years and if the maintenance cost was halved, the payback period would increase to 5.80 years. In addition, if the maintenance costs would be the same for the LED and fluorescent technology, this could change the payback period to 9.59 years.

The proposed lighting system validates that energy consumption may be reduced and energy saving is possible by using LED lamps. With safe energy, it is possible to improve diverse areas of the aircraft, making it more competitive and enhancing its value for the owner. Although the cost of purchasing the LED lamp combined with the project and installation costs imply a higher initial investment, in the long term, the returns obtained are worth the investment. The usage of LED lamps decreases the weight of the aircraft and the maintainability, which has a direct impact on the operational cost. Beyond that, the LED lamp brings an overall better passenger experience, since it provides a more comfortable environment for the passengers by making them feel less jet lag and apprehensive about the flight.

\section{AUTHORS' CONTRIBUTION}

Conceptualization: Guimarães IAB, Moraes AO and Barbosa DS; Methodology: Guimarães IAB, Moraes AO and Barbosa DS; Investigation: Guimarães IAB, Moraes AO and Barbosa DS; Writing - Original Draft: Guimarães IAB; Writing - Review and Editing: Moraes $\mathrm{AO}$ and Barbosa DS.

\section{DATA AVAILABILITY STATEMENT}

Data sharing is not applicable in this work.

\section{FUNDING}

Not applicable.

\section{ACKNOWLEDGEMENTS}

The first author thanks the support provided by Embraer's Programa de Especialização em Engenharia (PEE).

\section{REFERENCES}

Aeroflap (2018) Bombardier quer ganhar metade do mercado de aviação regional da Embraer. Aeroflap. [accessed Aug 10 2020]. https://www.aeroflap.com.br/bombardier-quer-ganhar-metade-do-mercado-de-aviacao-regional-da-embraer/

Airlines IATA (2014) Sponsored content: Embraer Commercial Aviation presents its new E-Jet cabin. Airlines IATA. [accessed Aug 5 2020]. https://airlines.iata.org/thought-leadership/sponsored-content-embraer-commercial-aviation-presents-its-new-e-jet-cabin 
Bachman J (2015) Airlines Add Mood Lighting to Chill Passengers Out. Bloomberg. [accessed Aug 5 2020]. https://www. bloomberg.com/news/articles/2015-04-22/airlines-add-mood-lighting-to-chill-passengers-out

Brodkey A (2014) Jetphotos. Embraer 170-200LR. [accessed Sep 10 2020]. https://www.jetphotos.com/photo/7820304

Clean Sky (2020) Regional Aircraft. [accessed Sep 20 2020]. https://www.cleansky.eu/regional-aircraft

Dilaura DL, Houser KW, Mistrick, RG, Steff, GR (2011) The Lighting Handbook: Reference and Application. New York: Illuminating Engineering Society.

Eden PE (2020) Embraer E-Jet E2 cabin: Evolutionary design. Aviation Business News. [accessed Jul 13 2020]. https://www. aviationbusinessnews.com/cabin/embraer-e-jet-e2-cabin-design-review/

EMBRAER (2020) E175. Commercial Aviation. Our Aircraft. [accessed Jul 112020$]$. https://www.embraercommercialaviation. com/commercial-jets/e175/

Escobar J (2005) Light Emitting Diodes: Gaining visibility in the aviation world. Aviationonpros. Aviation Pros. [accessed Aug 2 2020]. https://www.aviationpros.com/home/article/10385852/light-emitting-diodes-gaining-visibility-in-the-aviation-world

IflyA380 AIRBUS (2018) Seven Innovations That Make Your A380 Flight Super Smooth. IflyA380 AIRBUS. [accessed Aug 20 2019]. https://www.iflya380.com/fly-smart/Seven-Innovations-That-Make-Your-A380-Flight-Super-Smooth.html?origin=SJK

Markets and Markets (2020) Aircraft Lighting Market by Aircraft Type (Commercial, Military, Business Jets \& General Aviation, Helicopters), Light Source (LED, Fluorescent), Light Type (Interior Lights, Exterior Lights), End User (OEM, Aftermarket), Region-Global Forecast to 2030. Aircraft Lighting Market. Market Research Report. [accessed Aug 5 2020]. https://www.marketsandmarkets.com/Market-Reports/aircraft-lighting-market-1092.html

Nuhoglu EP (2016) Adaptive lighting: a research on interactive lighting design and technologies: the new lighting design project "LUVI" (Graduate thesis). Milan: Politecnico Di Milano.

OSRAM (2014) Manual Luminotécnico Prático. Osasco: OSRAM. [accessed Feb 10 2021]. https://www.fau.usp.br/arquivos/ disciplinas/au/aut0274/ilumART.\%20Manual\%20Osram\%20V1.pdf

Principi P, Fioretti R (2014) A comparative life cycle assessment of luminaires for general lighting for the office - compact fluorescent (CFL) vs Light Emitting Diode (LED) - a case study. Journal of Cleaner Production 83:96-107. https://doi. org/10.1016/j.jclepro.2014.07.031

Satco (2021) 3100 Lumens - 5 ft. T8 LED Tube - Ballast Bypass - 24 Watt - 4000 Kelvin. 1000 Bulbs. [accessed Jan 112021 ]. https://www.1000bulbs.com/product/216783/SATCO-S16406.html

Stawiarz L (2014) Jetphotos. 2014. 1 fotografia. [accessed Oct 28 2020]. https://www.jetphotos.com/photo/7951666

STG Aerospace (2017) Human Centric Lighting for Aircraft Passengers. STG Aerospace. [accessed Aug 20 2019]. https:// www.stgaerospace.com/application/files/2215/1914/5359/HCL_280317_Final_Print.pdf

Stone D (2017) The Hidden Costs of Flying. National Geographic. Urban Expeditions. [accessed Jul 10 2020]. https://www. nationalgeographic.com/environment/urban-expeditions/transportation/urban-expeditions-graphic-V21/

Sylvania (2021) Sylvania 21916 - FO40/841/XP/ECO3 - T8s. 1000Bulbs. [accessed Jan 11 2021]. https://www.1000bulbs.com/ product/191789/sylvania-216.html\#detail-tabs

Wynbrandt J (2016) NBAA Convention News. New Aircraft Lighting LEDs Replace Fluorescents. AINOnline. [accessed Aug 10 2019]. https://www.ainonline.com/aviation-news/business-aviation/2016-10-30/new-aircraft-lighting-leds-replace-fluorescents 